PROCEEDINGS OF THE

AMERICAN MATHEMATICAL SOCIETY

Volume 125, Number 5, May 1997, Pages 1407-1412

S 0002-9939(97)04016-

\title{
CHARACTERIZATIONS OF PSEUDODIFFERENTIAL OPERATORS ON THE CIRCLE
}

\author{
SEVERINO T. MELO
}

(Communicated by Palle E. T. Jorgensen)

\begin{abstract}
Globally defined operators are shown to be equivalent to the classical pseudodifferential operators on the circle. A characterization of the smooth operators for the regular representation of $\mathbb{S}^{1}$ is also given.
\end{abstract}

\section{INTRODUCTION}

The introduction of a symbol into the Fourier inversion formula on the circle defines a "discrete" pseudodifferential operator, as in the case of the pseudodifferential operators on $\mathbb{R}$. Several classes of these globally defined operators have been shown to be equivalent to classical pseudodifferential operators on $\mathbb{S}^{1}$, the first (unpublished) result of this kind being attributed by M. S. Agranovich [2] to L. R. Volevich. Theorems on this theme are found, for example, in the papers [1], [2], [11] and [8], where also many applications are developed or mentioned.

The smooth vectors for the canonical action of the Heisenberg group on $\mathcal{L}\left(L^{2}(\mathbb{R})\right)$, the algebra of bounded operators on $L^{2}(\mathbb{R})$, were characterized by H. O. Cordes [5] as those pseudodifferential operators whose symbols have bounded derivatives of all orders. Similar criteria hold for other Lie groups $[4,5]$. These results have been applied to nonlinear problems [9] and are also related to deformations of $C^{*}$-algebras [10].

In Section 2 of this paper, the discrete pseudodifferential operators are studied in detail. Theorem 2 is then a consequence of H. O. Cordes' smoothness theorem and of the equivalence between certain local pseudodifferential operators and a class of discrete pseudodifferential operators. The smooth vectors for the canonical action of the translation group on $\mathcal{L}\left(L^{2}\left(\mathbb{S}^{1}\right)\right)$ are thus characterized as those discrete pseudodifferential operators whose symbols have all derivatives bounded in $\mathbb{S}^{1}$, uniformly with respect to the discrete variable. This means, in particular, that the class $O p \psi_{d} t_{0}$ defined in Section 3 is a $\Psi^{*}$-algebra in the sense of B. Gramsch [6]. E. Schrohe's results [12] imply, in particular, that the class $O p \psi_{d} c_{0}$ defined in Section 2 is also a $\Psi^{*}$-algebra.

The present results can be extended to the $n$-dimensional torus with a few minor changes of definitions and notation. For the sake of transparency of the argument, however, only the case of $n=1$ is exposed. It must be pointed out that our

Received by the editors November 14, 1995

1991 Mathematics Subject Classification. Primary 47G30; Secondary 35S05, 58G15.

(C)1997 American Mathematical Society 
Theorem 1 is not completely original (see W. McLean's Theorem 4.4 of [8]), but its proof is somewhat simpler.

\section{Discrete PSEUdodiffERENTIAL OPERATORS}

The symbols considered here are of the form $b(x, j), x \in \mathbb{R}$ and $j \in \mathbb{Z}$, with $b(\cdot, j)$ being a $2 \pi$-periodic smooth function for each $j$. Denoting by $\nabla^{l}$ the $l$-th power of the difference operator acting on sequences, $(\nabla a)_{j}=a_{j+1}-a_{j}$, and by $\langle\cdot\rangle$ the function $\langle t\rangle=\sqrt{1+t^{2}}, t \in \mathbb{R}$, we say that $b$ is a discrete classical symbol of order $m$ if, for every pair of non-negative integers $(k, l)$, there is a constant $C_{k l}$ such that

$$
\left|\nabla^{l} \partial_{x}^{k} b(x, j)\right| \leq C_{k l}\langle j\rangle^{m-l}, j \in \mathbb{Z}
$$

The smallest $C_{k l}$ for which (1) holds is denoted by $\|b\|_{k l}$ and the class of all $b$ satisfying (1) is denoted by $\psi_{d} c_{m}$.

A discrete pseudodifferential operator $B$ acts on functions on the circle $\mathbb{S}^{1}$ and is defined by the formula

$$
B v\left(e^{i x}\right)=\sum_{j \in \mathbb{Z}} e^{i j x} b(x, j) v_{j}
$$

where $v_{j}$ denotes the Fourier coefficient $v_{j}=\frac{1}{2 \pi} \int_{-\pi}^{\pi} e^{-i j x} v\left(e^{i x}\right) d x$.

Proposition 1. If $b$ is a discrete classical symbol of order $m \in \mathbb{Z}$, then the equation (2) defines a continuous operator $B: C^{\infty}\left(\mathbb{S}^{1}\right) \rightarrow C^{\infty}\left(\mathbb{S}^{1}\right)$. If $m \leq 0$, then $B$ extends to a bounded operator on $L^{2}\left(\mathbb{S}^{1}\right)$ whose norm satisfies

$$
\|B\| \leq \max \left\{\|b\|_{2,0},\|b\|_{0,0}\right\} \cdot \sum_{j \in \mathbb{Z}}\langle j\rangle^{-2} .
$$

Proof. That $B$ is a well-defined continuous operator on $C^{\infty}\left(\mathbb{S}^{1}\right)$ follows from

$$
\sum_{j \in \mathbb{Z}}\left|j^{l} \partial_{x}^{k} b(x, j) v_{j}\right| \leq\|b\|_{k, 0} \cdot \sup \left|\left(1-\partial_{x}^{2}\right)^{p} v\left(e^{i x}\right)\right| \cdot \sum_{j \in \mathbb{Z}}\langle j\rangle^{m+l-2 p},
$$

where, for each $l, p$ is chosen so that $2 p-m-l \geq 2$.

In order to prove the $L^{2}$-boundedness, one needs to estimate the matrix elements $b_{k j}=(2 \pi)^{-1} \int_{-\pi}^{\pi} b(x, j) e^{i(j-k) x} d x$. Using that $e^{i(j-k) x}=\langle j-k\rangle^{-2}\left(1-\partial_{x}^{2}\right) e^{i(j-k) x}$ and integrating by parts, one gets $\left|b_{k j}\right| \leq \max \left\{\|b\|_{2,0},\|b\|_{0,0}\right\}\langle j-k\rangle^{-2}$. It follows that the sum $\sum_{j \in \mathbb{Z}}\left|b_{k j}\right|$ is bounded by the constant on the right-hand side of (3) for every $k$ and that the corresponding statement with the roles of $k$ and $j$ interchanged is also true. The proposition follows from Schur's lemma (see, for example, [3]).

The class of all operators $B$ with discrete classical symbols of order $m$ is denoted by $O p \psi_{d} c_{m}$. Next result states that it coincides with the usual class of pseudodifferential operators on the circle.

Theorem 1. An operator $B: C^{\infty}\left(\mathbb{S}^{1}\right) \rightarrow C^{\infty}\left(\mathbb{S}^{1}\right)$ belongs to Op $\psi_{d} c_{m}, m \in \mathbb{Z}$, if and only if it is a pseudodifferential operator of order $m$ on $\mathbb{S}^{1}$ in the usual sense; i.e., $B \in \Psi^{m}\left(\mathbb{S}^{1}\right)$ in the notation of [7], or $B \in L C_{m}\left(\mathbb{S}^{1}\right)$ in the notation of [4].

Proof. Let $B \in O p \psi_{d} c_{m}$ be given. For each $\theta \in \mathbb{R}$, let us consider the chart $\chi: \mathbb{S}^{1} \backslash\left\{-e^{i \theta}\right\} \rightarrow(-\pi+\theta, \pi+\theta), \chi\left(e^{i x}\right)=x$. Given $\varphi$ and $\omega$ in $C^{\infty}\left(\mathbb{S}^{1}\right)$, both vanishing on a neighborhood of $-e^{i \theta}$, let $B_{\theta}$ denote the operator on the Schwartz space $\mathcal{S}(\mathbb{R})$ defined by $\left(B_{\theta} u\right) \circ \chi=\omega B\left[\left(\varphi_{\theta} u\right) \circ \chi\right]$, where $\lambda_{\theta}=\lambda \circ \chi^{-1}$. In order 
to prove that $B \in \Psi^{m}\left(\mathbb{S}^{1}\right)$, it is enough to show that $B_{\theta}$ is a pseudodifferential operator on $\mathbb{R}$ whose symbol satisfies the estimates on (7).

The distribution kernel of $B_{\theta}$ as an operator on the Schwartz space $\mathcal{S}(\mathbb{R})$ equals

$$
\frac{1}{2 \pi} \sum_{j \in \mathbb{Z}} e^{i(x-y) j} b(x, j) \varphi_{\theta}(x) \omega_{\theta}(y)
$$

where the sum converges in distribution sense. If $B_{\theta}$ is a pseudodifferential operator on $\mathbb{R}$, its symbol must then be given by (see [4], Chapter 1 , for example)

$$
a(x, \xi)=\frac{\varphi_{\theta}(x)}{2 \pi} \sum_{j} b(x, j) \int e^{i(j-\xi) z} \omega_{\theta}(x-z) d z
$$

and conversely, if we show that this expression defines a proper symbol, then the equality $B_{\theta}=a(x, D)$ will be established.

The domain of the integral in (4) may be taken as the interval $[-2 \pi+\delta, 2 \pi-\delta]$, for some $\delta>0$, because the supports of $\varphi_{\theta}$ and of $\omega_{\theta}$ are both compact and contained in $(-\pi+\theta, \pi+\theta)$. The sum in equation (4) is absolutely convergent, since the integral appearing on the right-hand side is of the order of $\langle j-\xi\rangle^{-2 p}$, for any integer $p$. The derivatives with respect to $\xi$ may be brought inside the sum, since the absolute convergence also holds if we replace $\omega_{\theta}(x-z)$ by $z^{l} \omega_{\theta}(x-z), l \geq 1$. This power of $z$ and the use of the formula $e^{i j z}=\left(e^{i z}-1\right)^{-l} \nabla^{l} e^{i j z}$ allow us to do partial summation as follows:

$$
\begin{gathered}
\partial_{\xi}^{l} a(x, \xi)=\frac{\varphi_{\theta}(x)}{2 \pi} \sum_{j} b(x, j) \nabla^{l}\left(\int_{-2 \pi+\delta}^{2 \pi-\delta} e^{i(j-\xi) z} \frac{(-i z)^{l}}{\left(e^{i z}-1\right)^{l}} \omega_{\theta}(x-z) d z\right)= \\
(-1)^{l} \frac{\varphi_{\theta}(x)}{2 \pi} \sum_{j}\left[\nabla^{l} b(x, j-l)\right] \int_{-2 \pi-\delta}^{2 \pi+\delta} e^{i(j-\xi) z} \frac{(-i z)^{l}}{\left(e^{i z}-1\right)^{l}} \omega_{\theta}(x-z) d z .
\end{gathered}
$$

Using that $e^{i(j-\xi) z}=\langle j-\xi\rangle^{-2 p}\left(1-\partial_{z}^{2}\right)^{p} e^{i(j-\xi) z}$, integrating by parts and using also (1), it follows that

$$
\left|\partial_{\xi}^{l} a(x, \xi)\right| \leq C \sum_{j}\langle j\rangle^{m-l}\langle j-\xi\rangle^{-2 p}
$$

for any non-negative integer $p$, with $C$ equal to

$$
2\|b\|_{0, l} \sup _{j} \frac{\langle j-l\rangle^{m-l}}{\langle j\rangle^{m-l}} \sup _{|x-\theta|<\pi,|z|<2 \pi-\delta}\left|\varphi_{\theta}(x)\left(1-\partial_{z}^{2}\right)^{p} \frac{z^{l} \omega_{\theta}(x-z)}{\left(e^{i z}-1\right)^{l}}\right| .
$$

Using now that $\langle x\rangle \leq 2\langle y\rangle\langle x-y\rangle$, for all real $x$ and $y$, it follows that

$$
\left|\partial_{\xi}^{l} a(x, \xi)\right| \leq C 2^{|m-l|}\langle\xi\rangle^{m-l} \sum_{j \in \mathbb{Z}}\langle j-\xi\rangle^{|m-l|-2 p} .
$$

If $p$ is large enough, this last sum defines a continuous and periodic function of $\xi$ and is then bounded by a constant.

The previous argument also works after (4) is differentiated with respect to $x$. It then follows that $a$ is a classical symbol of order $m$, i.e., it satisfies the estimate

$$
\left|\partial_{x}^{k} \partial_{\xi}^{l} a(x, \xi)\right| \leq C_{k, l}\langle\xi\rangle^{m-l}
$$

Conversely, let $B \in \Psi^{m}\left(\mathbb{S}^{1}\right)$ be given. By means of a suitable choice of a partition of unity, it can be seen that it is enough to show that $B^{\dagger}=\varphi(M) B \omega(M)$ is in 
$O p \psi_{d} c_{m}$ for any pair $(\varphi, \omega)$ of functions in $C^{\infty}\left(\mathbb{S}^{1}\right)$ both vanishing on a neighborhood of some point $e^{-i \theta}$. We have denoted by $\zeta(M)$ the operator multiplication by $\zeta$. Since $B^{\dagger}$ is continuous on $C^{\infty}\left(\mathbb{S}^{1}\right)$, if it belongs to $O p \psi_{d} c_{m}$ its discrete symbol must then be given by

$$
b(x, j)=e^{-i j x} B^{\dagger} e_{j}\left(e^{i x}\right),
$$

where $e_{j}(z)=z^{j}, z \in \mathbb{S}^{1}$; and conversely, if equation (8) defines a discrete symbol in $\psi_{d} c_{m}$, the corresponding operator in $O p \psi_{d} c_{m}$ must then be equal to $B^{\dagger}$. All that is left is to show that $b$ defined on (8) indeed satisfies (1).

By hypothesis, $B^{\dagger}$ is locally given as a pseudodifferential operator on $\mathbb{R}$ with symbol $a$ satisfying (7). It then follows that

$$
b(x, j)=\frac{1}{2 \pi} \int_{-\infty}^{+\infty} a(x, \xi)\left(\int_{-\pi+\theta}^{\pi+\theta} e^{i(x-y)(\xi-j)} \rho(y) d y\right) d \xi
$$

where $\rho \in C_{0}^{\infty}(-\pi+\theta, \pi+\theta)$ is equal to one on the support of $\omega_{\theta}$. Using that $\nabla^{l} e^{i j z}=e^{i j z}\left(e^{i z}-1\right)^{l}$ and that $e^{i(x-y) \xi}=i^{-l}(x-y)^{-l} \partial_{\xi}^{l} e^{i(x-y) \xi}$, we get:

$$
\nabla^{l} b(x, j)=\frac{1}{2 \pi} \int_{-\infty}^{+\infty} a(x, \xi) \partial_{\xi}^{l}\left(\int_{-\pi+\theta}^{\pi+\theta} e^{i(x-y)(\xi-j)}\left[\frac{e^{i(y-x)}-1}{i(x-y)}\right]^{l} \rho(y) d y\right) d \xi
$$

In order to integrate by parts in $\xi$, we need the estimate

$$
\left|\partial_{\xi}^{q} \int_{-\pi+\theta}^{\pi+\theta} e^{i(x-y)(\xi-j)}\left[\frac{e^{i(y-x)}-1}{i(x-y)}\right]^{l} \rho(y) d y\right| \leq 2 \pi A_{p, l, q}(x)\langle\xi-j\rangle^{-2 p},
$$

which holds for any integers $p \geq 0$ and $0 \leq q \leq l$, with

$$
A_{p, l, q}(x)=\sup _{|y-\theta|<\pi}\left|\left(1-\partial_{y}^{2}\right)^{p}\left[\left(e^{i(y-x)}-1\right)^{l}(x-y)^{q-l} \rho(y)\right]\right| .
$$

If $p$ is chosen so that $m-l-2 p \leq-2$, then it follows from (7) that the desired integration by parts is legitimate and that

$$
\left|\nabla^{l} b(x, j)\right| \leq C_{0, l} A_{p, l, 0}(x) \int_{-\infty}^{+\infty}\langle\xi\rangle^{m-l}\langle\xi-j\rangle^{-2 p} d \xi .
$$

Since $A_{p, l, 0}$ depends continuously on $x$ and $b(x, j)$ is periodic in $x$, the inequality on (10) also holds with a constant independent of $x$. Using once more the inequality $\langle\xi\rangle^{m-l} \leq 2^{|m-l|}\langle j\rangle^{m-l}\langle\xi-j\rangle^{|m-l|}$ and requiring that $-2 p+|m-l| \leq-2$, it follows that the integral on the right-hand side of (10) is bounded by the constant $2^{|m-l|}\langle j\rangle^{m-l} \int\langle\xi\rangle^{-2} d \xi$. This proves (1) for $k=0$.

When (9) is differentiated with respect to $x$, some of the derivatives will fall on $a(x, \xi)$ and some on $e^{i(x-y)(\xi-j)}$. Those on the symbol cause no trouble, since any derivative of $a$ with respect to $x$ also satisfies (7) with different constants. Those on the exponential may be transformed into derivatives with respect to $y$, since $\partial_{x} e^{i(x-y)(\xi-j)}=-\partial_{y} e^{i(x-y)(\xi-j)}$. An integration by parts then brings the derivatives to $\rho$ and thus the same argument used for the case when $k=0$ will finish the proof. 


\section{SMOOTHNESS CRITERION}

Let us denote by $\psi_{d} t_{m}, m \in \mathbb{Z}$, the class of the symbols $b(x, j)$ satisfying (1) possibly only for $l=0$. Proposition 1 still holds for $b \in \psi_{d} t_{m}$, since only the finiteness of the seminorms $\|\cdot\|_{k, 0}, k \geq 0$, is needed in the proof. Denoting by $O p \psi_{d} t_{m}$ the class of all corresponding $B$ defined by (2), it is also clear that a weaker version of Theorem 1 is true; namely, that a continuous $B: C^{\infty}\left(\mathbb{S}^{1}\right) \rightarrow C^{\infty}\left(\mathbb{S}^{1}\right)$ belongs to $O p \psi_{d} t_{m}$ if and only if, for every real $\theta$ and every pair of smooth functions $(\varphi, \omega)$ vanishing on a neighborhood of $e^{-i \theta}$, the operator $B_{\theta}$ defined at the beginning of the proof of Theorem 1 is a pseudodifferential operator on $\mathbb{R}$ with symbol $a$ satisfying

$$
\left|\partial_{x}^{k} \partial_{\xi}^{l} a(x, \xi)\right| \leq C_{k, l}\langle\xi\rangle^{m} .
$$

Since the support of $a$ projects onto a compact subset of the $x$-axis, such a symbol belongs to $\psi t_{(m,-\infty)}$, as defined in [4].

Let $T_{z}, z \in \mathbb{R}$, denote the unitary operator on $L^{2}\left(\mathbb{S}^{1}\right)$ defined by $\left(T_{z} u\right)\left(e^{i x}\right)=$ $u\left(e^{i(x-z)}\right)$. The following is now a consequence of Theorem VIII.2.1 of [4].

Theorem 2. Given $B$ a bounded operator on $L^{2}\left(\mathbb{S}^{1}\right)$, the map

$$
\begin{aligned}
\mathbb{R} & \longrightarrow \mathcal{L}\left(L^{2}\left(\mathbb{S}^{1}\right)\right), \\
z & \longmapsto T_{z}^{-1} B T_{z}
\end{aligned}
$$

has operator-norm derivatives of all orders if and only if $B \in O p \psi_{d} t_{0}$.

Proof. If $B \in \mathcal{L}\left(L^{2}\left(\mathbb{S}^{1}\right)\right)$ is such that the map on (12) is smooth, the same is true for $\varphi(M) B \omega(M)$, where $\varphi$ and $\omega$ are smooth functions on $\mathbb{S}^{1}$. If both functions vanish on a neighborhood of some point, the operators $B(z)=T_{z}^{-1} \varphi(M) B \omega(M) T_{z}$ may be viewed as operators on $L^{2}(\mathbb{R})$, defining an operator-valued function which is norm-smooth with respect to $z$, for small $z \in \mathbb{R}$. We must show that conjugation of $B(z)$ by the multiplication operators $e^{i y M}$ is also $\mathcal{L}\left(L^{2}(\mathbb{R})\right)$-smooth with respect to $y$. Let $\varphi_{1}$ and $\omega_{1}$ be smooth functions of supports slightly larger than those of $\varphi$ and $\omega$ and identically equal to one on the supports of $\varphi$ and of $\omega$, respectively. We then have

$$
e^{i y M} B(0) e^{-i y M}=\left[e^{i y M} \varphi_{1}(M)\right] B(0)\left[e^{-i y M} \omega_{1}(M)\right],
$$

which is clearly smooth with respect to $y$. Using the commutation relation between $T_{z}$ and $e^{i y M}$, it follows that

$$
\begin{aligned}
\mathbb{R}^{2} & \longrightarrow \mathcal{L}\left(L^{2}(\mathbb{R})\right), \\
(z, y) & \longmapsto e^{-i y M} T_{-z}(\varphi(M) B \omega(M)) T_{z} e^{i y M}
\end{aligned}
$$

is norm smooth. Cordes' theorem quoted above then implies that $\varphi(M) B \omega(M)$ is locally given as a pseudodifferential operator on $\mathbb{R}$ with symbol $a \in \psi t_{(0,0)}$. It follows that $B$ is continuous on $C^{\infty}\left(\mathbb{S}^{1}\right)$ and thus that $B$ is in $O p \psi_{d} t_{0}$.

Conversely, a given $B \in O p \psi_{d} t_{0}$ can be written as a sum of operators of the type $\varphi(M) B \omega(M)$, with $\varphi$ and $\omega$ vanishing on a neighborhood of some point of the circle. Each of these operators is locally given as a pseudodifferential operator with symbol in $\psi t_{(0,0)}$. Again by Cordes' smoothness theorem, these local operators are smooth under the Heisenberg group action, in particular under the translation group action on $\mathcal{L}\left(L^{2}(\mathbb{R})\right)$. This shows that each of the operators $\varphi(M) B \omega(M)$ is $\mathcal{L}\left(L^{2}\left(\mathbb{S}^{1}\right)\right)$-smooth under the action of the translation group of $\mathbb{S}^{1}$. 


\section{ACKNOWLEDGEMENT}

The author thanks Prof. H. O. Cordes for suggesting the subject and for many helpful discussions.

\section{REFERENCES}

1. M. S. Agranovich, Spectral properties of elliptic pseudodifferential operators on a closed curve, Functional Anal. Appl. 13 (1979), 279-281. MR 81e:35096

2. M. S. Agranovich, On elliptic pseudodifferential operators on a closed curve, Trans. Moscow Math. Soc. 47 (1985), 23-74.

3. H. O. Cordes, Elliptic Pseudodifferential Operators - An Abstract Theory, Lecture Notes in Math. 756, 1979. MR 81j:47041

4. H. O. Cordes, The technique of pseudodifferential operators, Cambridge Univ. Press, 1995. MR 96b:35001

5. H. O. Cordes, On pseudodifferential operators and smoothness of special Lie-group representations, Manuscripta Math. 28 (1979), 51-69. MR 80m:47047

6. B. Gramsch, Relative Inversion in der Störungstheorie von Operatoren und $\Psi$-Algebren, Math. Ann. 269 (1984), 27-71. MR 86j:47065

7. L. Hörmander, The Analysis of Linear Differential Operators III, Springer, 1985. MR 87d:35002a

8. W. McLean, Local and global descriptions of periodic pseudodifferential operators., Math. Nach. 150 (1991), 151-161. MR 92f:47055

9. K. Payne, Smooth Tame Fréchet Algebras and Lie Groups of Pseudodifferential Operators, Comm. Pure Appl. Math. 44 (1991), 309-337. MR 92a:58140

10. M. Rieffel, Deformation Quantization for actions of $\mathbf{R}^{d}$, Mem. Amer. Math. Soc. 506, 1993. MR 94d: 46072

11. J. Saranen and W. L. Wendland, The Fourier series representation of pseudodifferential operators on closed curves, Complex Variables Theory Appl. 8 (1987), 55-64. MR 88i:47028

12. E. Schrohe, $A \Psi^{*}$-algebra of pseudodifferential operators on noncompact manifolds, Arch. Math. 51 (1988), 81-86. MR 89i:47092

Instituto de Matemática e Estatística, Universidade de São Paulo, Caixa Postal 66281, SÃo PAULO 05315-970, BRAZIL

E-mail address: toscano@ime.usp.br 